\title{
Hepatotoxicidad por isoniazida en un paciente con enfermedad de Crohn. Reporte de caso y revisión de la literatura
}

\section{Hepatotoxicity due to Isoniazide in a Patient with Crohn's Disease: Case Report and Literature Review}

Gustavo Adolfo Reyes M., MD, ${ }^{1}$ Germán David Carvajal P., MD, ${ }^{2}$ Mónica Lorena Tapias M., MD, ${ }^{3}$ Luis Carlos Sabbagh S., MD. ${ }^{4}$

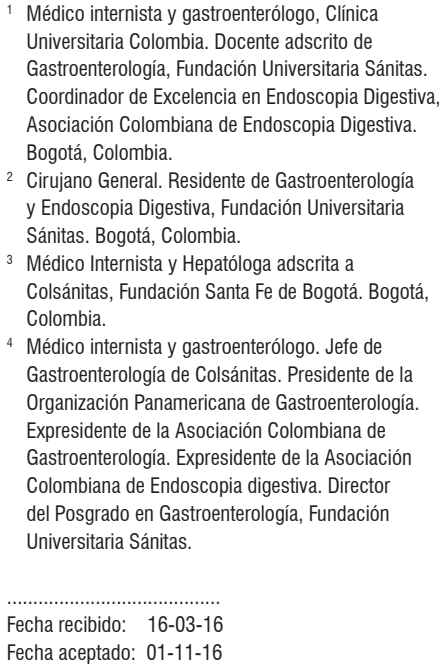

Médico internista y gastroenterólogo, Clínica Universitaria Colombia. Docente adscrito de Gastroenterología, Fundación Universitaria Sánitas. Coordinador de Excelencia en Endoscopia Digestiva, Asociación Colombiana de Endoscopia Digestiva. Bogotá, Colombia.

2 Cirujano General. Residente de Gastroenterología y Endoscopia Digestiva, Fundación Universitaria Sánitas. Bogotá, Colombia

${ }^{3}$ Médico Internista y Hepatóloga adscrita a Colsánitas, Fundación Santa Fe de Bogotá. Bogotá, Colombia.

${ }^{4}$ Médico internista y gastroenterólogo. Jefe de Gastroenterología de Colsánitas. Presidente de la Organización Panamericana de Gastroenterología. Expresidente de la Asociación Colombiana de Gastroenterología. Expresidente de la Asociación Colombiana de Endoscopia digestiva. Director del Posgrado en Gastroenterología, Fundación Universitaria Sánitas.

Fecha recibido: 16-03-16 Fecha aceptado: 01-11-16

\begin{abstract}
Resumen
La isoniazida se utiliza para el tratamiento o profilaxis de la tuberculosis; sin embargo, su uso puede asociarse con reacciones hepáticas adversas. La hepatitis clínicamente manifiesta sucede en $0,5 \%-1 \%$ de los pacientes que reciben isoniazida como monoterapia. En este artículo se describe el caso de un paciente con enfermedad de Crohn que cursó con hepatotoxicidad grave por isoniazida, y se hace una revisión de la literatura al respecto.
\end{abstract}

\section{Palabras clave}

Isoniazida, hepatotoxicidad, DILI (idiosyncratic drug-induced liver injury), enfermedad de Crohn.

\section{Abstract}

Isoniazid is used for treatment or prophylaxis of tuberculosis but may be associated with adverse hepatic reactions. Clinically manifest hepatitis occurs in $0.5 \%-1 \%$ of patients who receive isoniazid as monotherapy. This article describes the case of a patient with Crohn's disease who experienced severe hepatotoxicity due to isoniazid. It also reviews the literature.

Keywords

Isoniazid, hepatotoxicity, DILI (idiosyncratic drug-induced liver injury), Crohn's disease.

\section{INTRODUCCIÓN}

La lesión hepática idiosincrática por medicamentos (DILI, por sus siglas en inglés para Idiosyncratic Drug-induced Liver Injury) se caracteriza por una alteración en el perfil bioquímico hepático como reacción adversa a un medicamento utilizado en dosis apropiadas para la profilaxis o tratamiento de una enfermedad (1).

Su presentación clínica tiene un amplio espectro, desde una elevación asintomática, casi siempre autolimitada, del perfil bioquímico hepático, hasta ictericia e insuficiencia hepática aguda potencialmente mortal. Con poca frecuen- cia, puede causar enfermedad hepática crónica (2). Su incidencia varía entre 1:10 000 a 1:100 000 casos (3).

$\mathrm{La}$ idiosincrasia se refiere a diferencias individuales en la respuesta a un estímulo, en este caso a un medicamento, debido a factores genéticos y ambientales (4). En la India, los medicamentos antituberculosos son la principal causa de insuficiencia hepática aguda; mientras que en los países occidentales, la principal causa es el acetaminofén, seguida por los antimicrobianos $(5,6)$.

La fisiopatología de la DILI no es completamente entendida. Se propone la hipótesis del estrés inflamatorio, en donde los lipopolisacáridos bacterianos, liberados como 
resultado de una reacción inflamatoria, actúan en conjunto con los metabolitos del medicamento para causar la DILI. Otros factores planteados son el daño mitocondrial y la autoinmunidad (7). Como factores de riesgo están la edad ( $>55$ años), sexo (femenino), alcohol, polifarmacia, desnutrición, VIH, hepatitis viral B, C y factores genéticos $(4,7)$.

Desde el punto de vista histopatológico, la DILI se manifiesta como daño hepático agudo con diferentes patrones: lesión hepatocelular aguda, esteatosis aguda, colestasis aguda y patrón mixto colestásico-necroinflamatorio (8).

\section{CASO CLÍNICO}

Hombre de 64 años, con enfermedad de Crohn ileocolónica desde hace 10 años. Por intolerancia a inmunomoduladores, suspendidos 9 meses atrás, y enfermedad clínica y endoscópicamente activa, es candidato a terapia biológica. El paciente negó consumo de alcohol y terminó ciclo de budesónida hace 2 meses. El perfil bioquímico hepático inicial fue normal, la serología de hepatitis B y C fue negativa (tabla 1) y la PPD fue positiva 12 milímetros (normal hasta 5 milímetros). Se indicó profilaxis contra tuberculosis latente.

Inició $300 \mathrm{mg} /$ día de isoniazida por vía oral. A los 46 días, presentó astenia, adinamia, coluria, acolia, ictericia y náuseas. Consultó a Urgencias donde encontraron a un paciente en buenas condiciones generales, con signos vitales estables, ictericia marcada, leve dolor a la palpación profunda en hipocondrio derecho, sin signos de irritación peritoneal y sin alteración neurológica. Los paraclínicos (tabla 2) mostraron aminotransferasas elevadas $>10$ veces e hiperbilirrubinemia a expensas de la directa, sin signos clínicos o paraclínicos de insuficiencia hepática. Ecografía hepatobiliar normal. Estudios de hepatitis viral negativos.

Se ordenó suspender la isoniazida y se dio de alta con diagnóstico de hepatitis en estudio sin insuficiencia hepática. Evolucionó satisfactoriamente con mejoría de los síntomas y desaparición de la ictericia 2 semanas después del alta. El paciente reinició la isoniazida y 3 días después presentó nuevamente ictericia, coluria, astenia y adinamia, por lo que suspendió el medicamento y consultó nuevamente por Urgencias, donde encuentran a un paciente en buenas condiciones generales, con signos vitales normales, ictérico, examen abdominal y neurológico normales, los paraclínicos (tabla 3) muestran nuevamente aminotransferasas $>10 x$ e hiperbilirrubinemia marcada, PT y glicemia normales, ecografía hepatobiliar normal.

Evolucionó satisfactoriamente y se dio de alta con indicación de no iniciar ningún medicamento hasta nueva orden médica y controles ambulatorios semanales, en los cuales se observó mejoría del cuadro clínico y disminución progresiva hasta normalización del perfil bioquímico hepático, estudio de hepatitis autoinmune y hemocromatosis negativos (tabla
4). En las figuras 1 y 2 se grafica la evolución de las aminotransferasas y de las bilirrubinas durante el cuadro clínico.

Tabla 1. Exámenes de laboratorio iniciales, antes de la administración de isoniazida

\begin{tabular}{lc}
\hline \multicolumn{1}{c}{ Examen de laboratorio } & Valor \\
\hline ALT & $12 \mathrm{U} / \mathrm{L}$ \\
AST & $12 \mathrm{U} / \mathrm{L}$ \\
Bilirrubina total & $0,7 \mathrm{mg} / \mathrm{dL}$ \\
Bilirrubina directa & $0,35 \mathrm{mg} / \mathrm{dL}$ \\
Bilirrubina indirecta & $0,35 \mathrm{mg} / \mathrm{dL}$ \\
Fosfatasa alcalina & $90 \mathrm{U} / \mathrm{L}$ \\
Anticuerpos contra antígeno de superficie, & Negativo \\
hepatitis B, anticuerpos anticore total & \\
Antígeno de superficie de hepatitis B & Negativo \\
Anticuerpos contra hepatitis C & Negativo \\
\hline
\end{tabular}

Tabla 2. Exámenes de laboratorio el día 46

\begin{tabular}{lc}
\hline$\quad$ Examen de laboratorio & Valor \\
\hline ALT & $1826 \mathrm{U} / \mathrm{L}$ \\
AST & $1130 \mathrm{U} / \mathrm{L}$ \\
Fosfatasa alcalina & $155 \mathrm{U} / \mathrm{L}$ \\
Bilirrubina total & $17 \mathrm{mg} / \mathrm{dL}$ \\
Bilirrubina directa & $14 \mathrm{mg} / \mathrm{dL}$ \\
Tiempo de protrombina & $10,2 \mathrm{~s}$ \\
INR & 1,0 \\
Glucosa & $76 \mathrm{mg} / \mathrm{dL}$ \\
\hline
\end{tabular}

Tabla 3. Exámenes de laboratorio el día 60

\begin{tabular}{lc}
\hline$\quad$ Exámenes de laboratorio & Valor \\
\hline ALT & $854 \mathrm{U} / \mathrm{L}$ \\
AST & $542 \mathrm{U} / \mathrm{L}$ \\
Fosfatasa alcalina & $117 \mathrm{U} / \mathrm{L}$ \\
Bilirrubina total & $12 \mathrm{mg} / \mathrm{dL}$ \\
Bilirrubina directa & $10 \mathrm{mg} / \mathrm{dL}$ \\
Tiempo de protrombina & $13 \mathrm{~s}$ \\
\hline
\end{tabular}

Tabla 4. Exámenes de laboratorio del día 104

\begin{tabular}{lc}
\hline$\quad$ Exámenes de laboratorio & Valor \\
\hline ALT & $11 \mathrm{U} / \mathrm{L}$ \\
AST & $14 \mathrm{U} / \mathrm{L}$ \\
Fosfatasa alcalina & $32 \mathrm{U} / \mathrm{L}$ \\
Bilirrubina total & $0,8 \mathrm{mg} / \mathrm{dL}$ \\
Bilirrubina directa & $0,34 \mathrm{mg} / \mathrm{dL}$ \\
\hline
\end{tabular}

El paciente recibió profilaxis para tuberculosis con rifampicina, la cual toleró bien. Luego, inicia adalimumab; pre- 


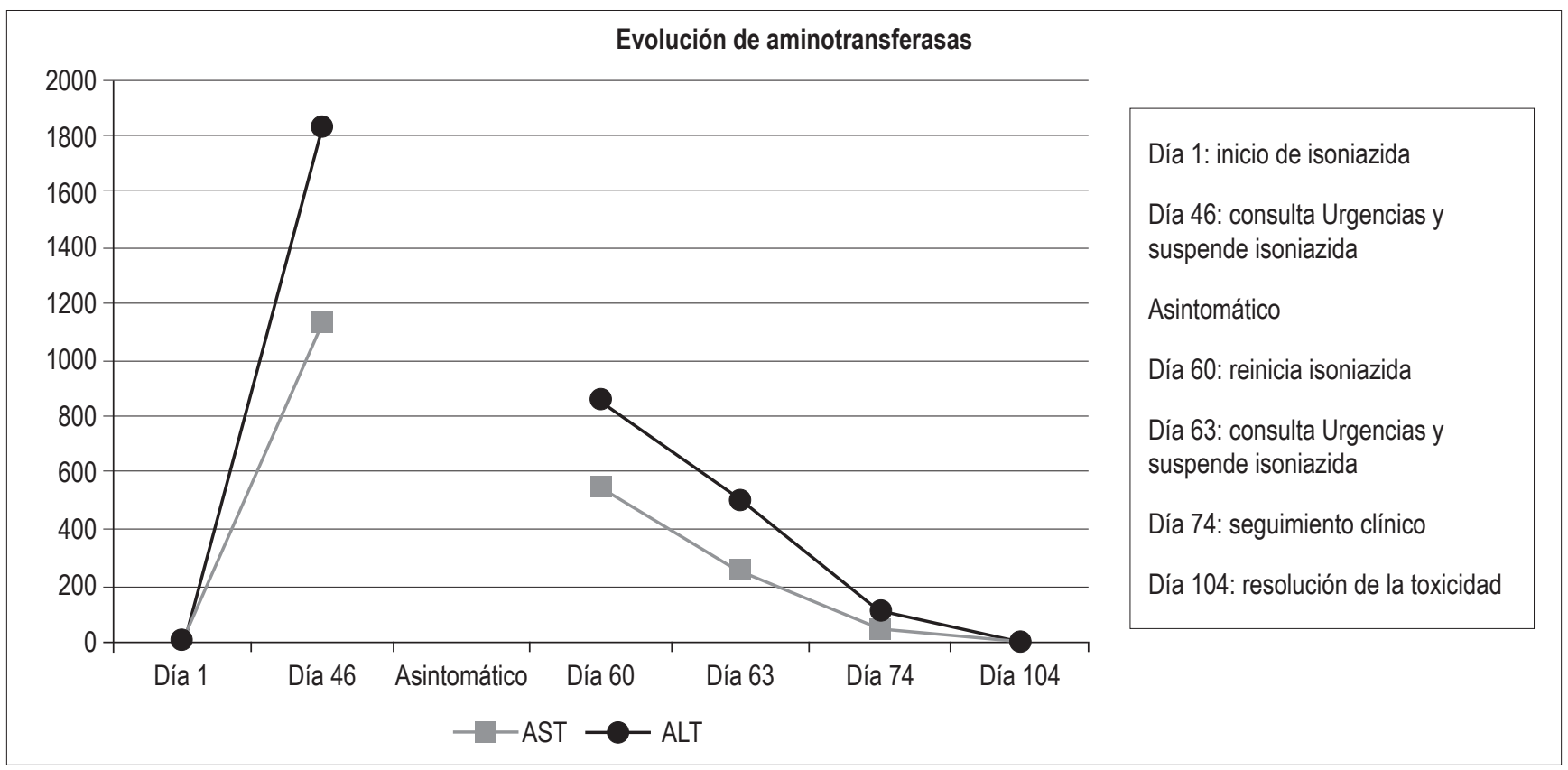

Figura 1. Evolución de aminotransferasas.

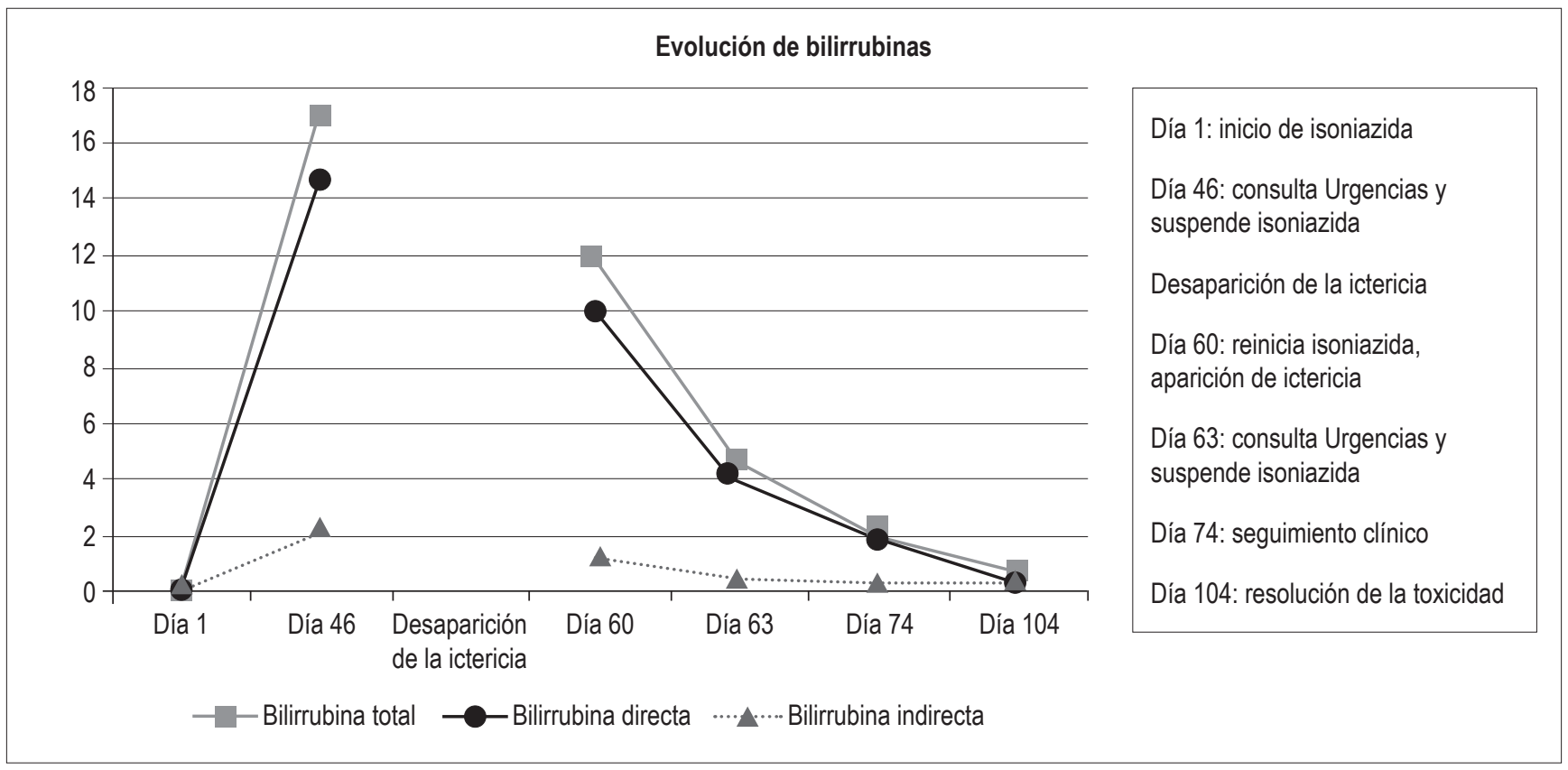

Figura 2. Evolución de bilirrubinas.

sentó mejoría clínica y remisión de los síntomas ocasionados por la enfermedad de Crohn. Al momento de escribir este artículo, el paciente lleva 1 año sin presentar alteración del perfil bioquímico hepático.

\section{DISCUSIÓN}

La DILI es un diagnóstico de exclusión (tabla 5), siempre deben descartarse otras causas más frecuentes de lesión 
hepática como hepatitis infecciosa, autoinmune, NASH, obstrucción biliar, lesiones vasculares, neoplasias hepáticas, enfermedad de Wilson o hemocromatosis.

Tabla 5. Criterios de diagnósticos para DILI (7)

Elevación de ALT y/o AST > 5 veces (paciente asintomático)

Aumento de la fosfatasa alcalina y/o bilirrubina total $>2$ veces asociado con cualquier incremento de las aminotransferasas

Cualquier elevación de aminotransferasas asociada con los síntomas

El período de latencia entre la exposición al medicamento y el desarrollo de DILI varía entre 5 a 90 días. $\mathrm{Al}$ eliminar el medicamento sospechoso, las aminotransferasas deben disminuir por lo menos $50 \%$ en los siguientes 8 días. La reexposición al medicamento como confirmación diagnóstica se puede considerar poco ético, excepto cuando no hay otra opción terapéutica. Casos informados de reexposición involuntaria confirman que un determinado medicamento es hepatotóxico. La sola elevación de aminotransferasas o fosfatasa alcalina, sin hiperbilirrubinemia o ictericia, se considera como enfermedad leve. La ictericia o hiperbilirrubinemia $>2 \mathrm{mg} / \mathrm{dL}$ indica enfermedad grave, como en el caso que se presenta. Si existe ictericia asociada con INR $>1,5$, encefalopatía o ascitis, el riesgo de mortalidad es hasta $21 \%$ en el caso de DILI por medicamentos antituberculosos (7).

La isoniazida se utiliza como terapia combinada para el manejo de la tuberculosis activa o como monoterapia para la tuberculosis latente. La isoniazida puede causar reacciones adversas hepáticas que se manifiestan en fases tempranas del tratamiento, desde 1 semana hasta 6 meses, como una hepatitis leve (10\%-20\% de los pacientes), típicamente asintomática y autolimitada, con elevación de ALT <3 veces (9). Generalmente, la ALT se normaliza a pesar de continuar el tratamiento, este fenómeno se denomina adaptación (10). Otra forma de presentación es la hepatitis sintomática $(0,5 \%-1 \%)$ asociada con síntomas gastrointestinales, ictericia y riesgo de progresión a insuficiencia hepática $(0,01 \%)(9,11)$.

Como factores de riesgo se encuentran: edad mayor de 35 años, sexo femenino, fenotipo de acetilador lento, tratamiento concomitante con otros medicamentos antiTBC o acetaminofén, dosis diaria de isoniazida mayor de $50 \mathrm{mg} /$ $\mathrm{kg}$, consumo de etanol y genotipo del citocromo P-450 2E1 $\mathrm{cl} / \mathrm{c} 1(12)$.

Los mecanismos propuestos de hepatotoxicidad por isoniazida son (12-14):

- Generación de metabolitos tóxicos: acetilhidrazina e hidracina, generadas por el metabolismo hepático de la isoniazida, se consideran hepatotóxicas. Los aceti- ladores lentos acumulan una mayor cantidad de estos metabolitos.

- Genotipo del citocromo P450 2E1 c1/c1: la mayor actividad de este citocromo produce más cantidades de metabolitos hepatotóxicos.

- Estrés oxidativo: la isoniazida y su metabolito hidracina disminuyen los niveles de glutatión y la actividad de la glutatión-S-transferasa (antioxidantes), lo que genera un aumento de los mecanismos oxidantes y, por ende, de estrés oxidativo.

- Respuesta inmune inducida por la isoniazida: se encontraron anticuerpos dirigidos contra isoniazida y el citocromo $\mathrm{P} 450$ en pacientes con insuficiencia hepática originada por el medicamento.

\section{CONCLUSIONES}

Se presenta el caso de un paciente de 64 años con hepatotoxicidad idiosincrática grave por isoniazida (hepatitis aguda-hiperbilirrubinemia marcada) la cual se confirmó debido a que existió una mejoría al suspenderla y luego reactivación por reexposición involuntaria al medicamento (no indicada por el médico), con evolución adecuada después de suspender el medicamento. Debido al incremento en la incidencia de enfermedad inflamatoria intestinal en nuestro medio, en el cual existe una alta exposición a la tuberculosis, los médicos se enfrentan cada vez con mayor frecuencia a la necesidad de suministrar profilaxis antituberculosis previo al uso de medicamentos biológicos, por lo cual, es importante tener en cuenta la posibilidad de hepatotoxicidad por isoniazida y realizar el seguimiento adecuado en estos pacientes.

\section{Financiación}

Ninguna.

\section{Conflictos de interés}

Ninguno.

\section{REFERENCIAS}

1. Edwards RI, Aronson JK. Adverse drug reactions: definition, diagnosis and management. Lancet. 2000;356:1255-9.

2. Navarro VJ, Senior JR. Drug related hepatotoxicity. N Eng J Med. 2006;354:731-9.

3. Chalasani N, Fontana RJ, Bonkovsky HL, et al. Causes, clinical features, and outcomes from a prospective study of druginduced liver injury in the United States. Gastroenterology. 2008;135:1924-34. 
4. Stirnimann G, Kessebohm K, Lauterburg B. Liver injury caused by drugs: an update. Swiss Med Wkly. 2010;140:w13080.

5. Kumar R, Shalimar, Bhatia B, et al. Antituberculosis therapyinduced acute liver failure: magnitude, profile, prognosis, and predictors of outcome. Hepatology. 2010;51:1655-74.

6. Reuben A, Koch DG, Lee WM. Drug-induced acute liver failure: results of a U.S. multicenter, prospective study. Hepatology. 2010;52:2065-76.

7. Devarbhavi H. An update on drug-induced liver injury. J Clin Exp Hepatol. 2012;2:247-59.

8. López R. Aspectos morfológicos de la enfermedad hepática inducida por drogas. Rev Col Gastroentero. 2014;29(4):449-60.

9. James L, Roberts D. Isoniazid hepatotoxicity: progress in understanding the immunologic component. Editorials Hepatology. 2014;59(3):746-8.
10. Watkins PB. Idiosyncratic liver injury: challenges and approaches. Toxicol Pathol. 2005;33:1-5.

11. Devarbhavi H, Andrade RJ. Drug-induced liver injury due to antimicrobials, central nervous system agents, and nonsteroidal anti-inflammatory drugs. Semin Liver Dis. 2014;34:145-61.

12. Metushi IG, Cai P, Zhu X, et al. A fresh look at the mechanism of isoniazid-induced hepatotoxicity. Clin Pharmacol Ther. 2011;89:911-4.

13. Tostmann A, Boeree MJ, Aarnoutse RE, et al. Antituberculosis drug-induced hepatotoxicity: concise up-to-date review. J Gastroenterol Hepatol. 2008;23(2):192-202.

14. Metushi IG, Sanders C, The Acute Liver Study Group, et al. Detection of anti-isoniazid and anti-cytochrome $\mathrm{p} 450$ antibodies in patients with isoniazid-induced liver failure. Hepatology. 2014;59(3):1084-93. 